\title{
Superoxide Radical Dismutation as New Therapeutic Strategy in Parkinson's Disease
}

\author{
Federica De Lazzari' ${ }^{1}$, Luigi Bubacco ${ }^{1}$, Alexander J Whitworth ${ }^{2}$, Marco Bisaglia ${ }^{1, *}$ \\ ${ }^{1}$ Molecular Physiology and Biophysics Unit, Department of Biology, University of Padova, 35131 Padova, Italy. \\ ${ }^{2}$ Medical Research Council Mitochondrial Biology Unit, University of Cambridge, Cambridge Biomedical \\ Campus, Cambridge, CB2 0XY, UK.
}

[Received July 11, 2017; Revised October 10, 2017; Accepted October 18, 2017]

\begin{abstract}
Aging is the biggest risk factor for developing many neurodegenerative disorders, including idiopathic Parkinson's disease (PD). PD is still an incurable disorder and the available medications are mainly directed to the treatment of symptoms in order to improve the quality of life. Oxidative injury has been identified as one of the principal factors involved in the progression of PD and several indications are now reported in the literature highlighting the prominent role of the superoxide radical in inducing neuronal toxicity. It follows that superoxide anions represent potential cellular targets for new drugs offering a novel therapeutic approach to cope with the progression of the disease. In this review we first present a comprehensive overview of the most common cellular reactive oxygen and nitrogen species, describing their cellular sources, their potential physiological roles in cell signalling pathways and the mechanisms through which they could contribute to the oxidative damage. We then analyse the potential therapeutic use of SOD-mimetic molecules, which can selectively remove superoxide radicals in a catalytic way, focusing on the classes of molecules that have therapeutically exploitable properties.
\end{abstract}

Key words: antioxidants, oxidative damage, Parkinson's disease, SOD-mimetics, superoxide dismutases

Parkinson's Disease (PD) represents a common neurodegenerative disorder that affects approximately 1 $2 \%$ of population over the age of 65 years. The parkinsonian symptoms include bradykinesia, resting tremors, muscular rigidity, postural instability, and gait impairment [1]. The neuropathology of PD is characterized by a preferential neuronal loss of dopaminergic cells within Substantia Nigra pars compacta (SNpc) and by the presence of inclusions called Lewy bodies in the surviving neurons [2]. Although most PD cases are sporadic, the identification of familial forms of the syndrome, accounting for 5-10\% of PD cases, has helped to shed some light on the cellular pathways involved in the pathology. Genetic manifestations of the disease are classified into dominant or recessive forms, according to the pattern of inheritance. $\alpha$-Synuclein and LRRK2 contribute to the former, while Parkin, PINK1, and DJ-1 are associated to the latter. $\alpha$-Synuclein is a major constituent of Lewy Bodies aggregates, while LRRK2 is a protein kinase involved in multiple cellular processes, including neurite outgrowth and synaptic morphogenesis. In contrast, Parkin, PINK1, and DJ-1 are all primarily implicated in mitochondrial health. Parkin, an E3 ubiquitin ligase, and PINK1, a serine-threonine protein kinase, cooperate to remove damaged mitochondria, whilst DJ-1 is redox-sensitive protein which protects mitochondria and cells against oxidative stress via an unknown mechanism [1]. Even though the aetiology of sporadic PD remains elusive, there is evidence that oxidative injury plays a significant role in

*Correspondence should be addressed to: Dr. Marco Bisaglia, Department of Biology, University of Padova, Via Ugo Bassi 58/B, 35131 Padova, Italy. Email: marco.bisaglia@unipd.it

Copyright: (C) 2017 De Lazzari F et al. This is an open-access article distributed under the terms of the Creative Commons Attribution License, which permits unrestricted use, distribution, and reproduction in any medium, provided the original author and source are credited. 
the development of the disease [3,4]. Notwithstanding the intense research performed in the field, at present there is no cure for PD, but only symptomatic treatment, principally based on the administration of L-DOPA, the dopamine precursor [5].

\section{Oxidative Injury and PD}

A special interest has been attributed to the role of oxidative damage in neurodegeneration, since the discovery that SN in PD patients presents high levels of oxidized DNA, proteins and lipids and lower levels of glutathione (GSH) [6], suggesting that the excessive production of reactive oxygen species (ROS) and reactive nitrogen species (RNS) might contribute to neuronal death. Oxidative injury derives from an imbalance in ROS/RNS homeostasis, due to an excessive production of these species or impairment in their detoxification. As represented in Fig. 1, multiple causes seem to trigger oxidative damage in PD. Indeed, mitochondrial dysfunction, dopamine metabolism and neuroinflammation are all thought to be involved in this stressful condition [3].

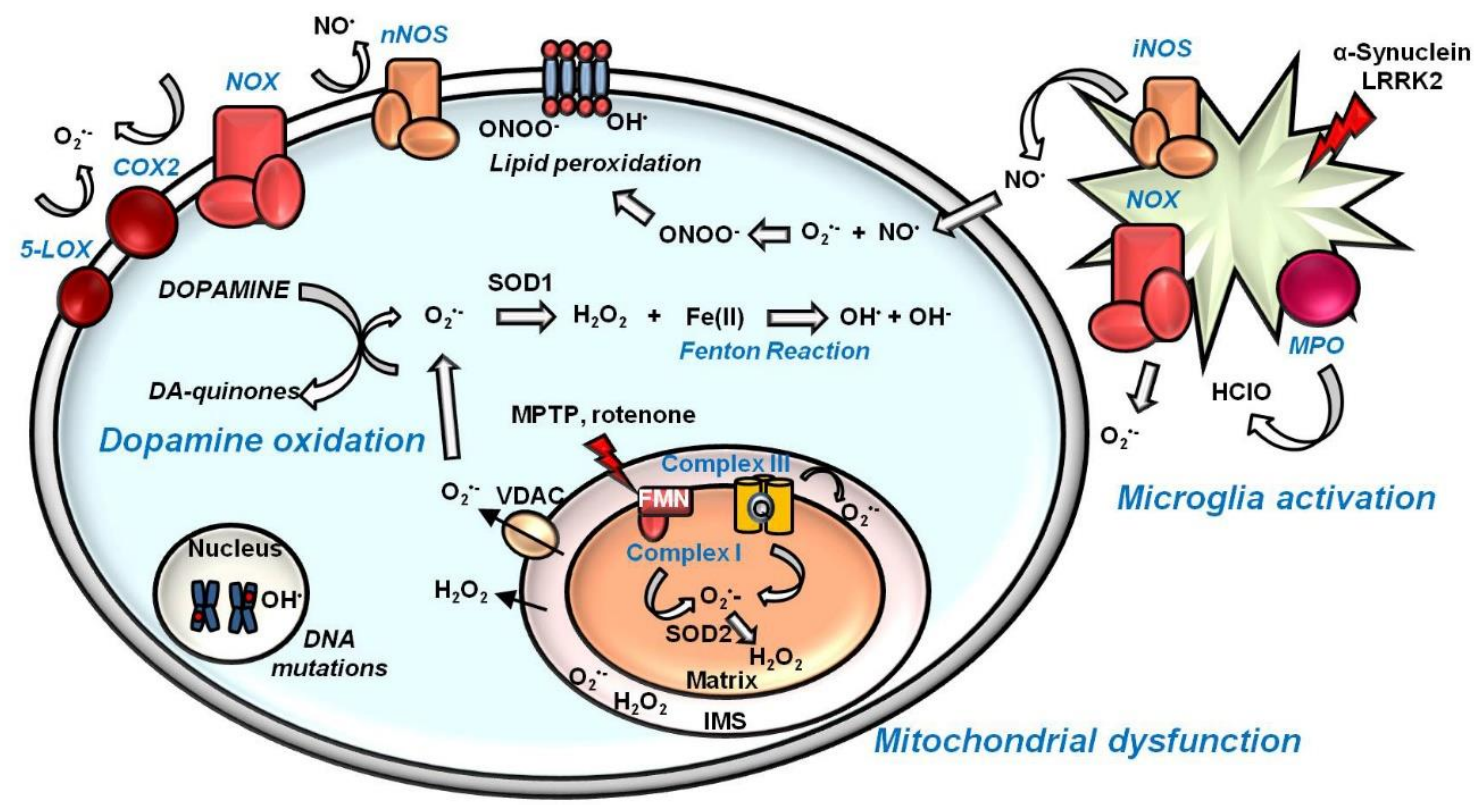

Figure 1. Oxidative injury and PD. Mitochondrial dysfunction, dopamine metabolism and neuroinflammation co-participate in promoting oxidative damage in dopaminergic neurons. Complexes I and III of the mitochondrial electron transport chain are the main sources of $\mathrm{O}_{2}{ }^{-}$inside cells. The PD-related toxins MPTP and rotenone are complex I inhibitors. Chronic activation of microglia represents a further mechanism of $\mathrm{O}_{2}{ }^{-}$- and $\mathrm{NO}^{*}$ radical's generation through the action of NADPH-oxidase (NOX) and inducible nitric oxide synthase (iNOS), respectively. In activated microglia, myeloperoxidase (MPO) is responsible for the formation of HClO. The PD-associated proteins $\alpha$-Synuclein and LRRK2 contribute to microglia activation. Specific to dopaminergic neurons, the cytosolic oxidation of dopamine to dopamine-quinones leads to the production of the $\mathrm{O}_{2}{ }^{*}$. Cytosolic $\mathrm{O}_{2}{ }^{*-}$ is transformed by the action of superoxide dismutase 1 (SOD1) in $\mathrm{H}_{2} \mathrm{O}_{2}$, which can produce the highly toxic radical $\mathrm{HO}^{\bullet}$ through the Fenton reaction. Otherwise, $\mathrm{O}_{2}{ }^{*}$ can react with $\mathrm{NO}^{*}$ to form the very reactive molecule $\mathrm{ONOO}^{-}$. (FMN: flavin mononucleotide; IMS: intermembrane space; nNOS: neuronal nitric oxide synthase; VDAC: voltage-dependent anion channels).

\section{Mitochondrial dysfunction}

Because of its high-energy demand, brain functions strongly depend on mitochondrial activity. Even when the mitochondrial electron transport chain (ETC) works efficiently, the redox reactions that occur during oxidative phosphorylation render each electron carrier in the ETC prone to react with molecular oxygen, producing ROS [7].
Superoxide anion $\left(\mathrm{O}_{2}{ }^{*-}\right)$ and hydrogen peroxide $\left(\mathrm{H}_{2} \mathrm{O}_{2}\right)$ represent the major ROS species produced during oxidative phosphorylation, and their generation is substantially increased when ETC is impaired in dysfunctional mitochondria. The discovery that MPTP (1methyl-4-phenyl-1,2,3,6-tetrahydropyridine), a contaminant of a synthetic opiate, can cause parkinsonism through its neurotoxic metabolite, 1-methyl-4- 
phenylpyridinium (MPP+), which acts as a complex I inhibitor, revealed a role of mitochondria in PD pathology [8]. Successively, other inhibitors such as rotenone, pyridaben, and fenpyroximate have been found, supporting the role of mitochondrial complex I dysfunction in oxidative stress [9]. Accordingly, postmortem analysis on PD patients showed that mitochondria frequently present a lowered complex I activity [10-12]. Besides complex I, also complex II and III can participate in ROS generation, since their components can promote oxygen reduction, leading to $\mathrm{O}_{2}{ }^{-}$[7]. Further support to the involvement of mitochondria in $\mathrm{PD}$ and ROS production derives from the finding that the PD-associate proteins Parkin, PINK1 and DJ-1 are involved in mitochondrial functioning. Studies in fruit flies clearly demonstrated that PINK1 and Parkin participate in controlling mitochondrial morphology and homeostasis [13-15]. Moreover, it has been reported that overexpressing DJ-1 pathological mutants in neuronal cells results in mitochondria fragmentation [16], and that, under oxidative conditions, DJ-1 translocates into mitochondria where it exerts its protective function, promoting cell survival [17]. Interestingly, DJ-1 has been reported to directly bind subunits of the mitochondrial complex I, regulating its activity [18]. In conclusion, mitochondria are critical organelles for the maintenance of brain functionality and their dysfunction seems to be deeply connected to increased oxidative conditions, which, in turn, might render dopaminergic neurons highly susceptible to cell death.

\section{Neuroinflammation}

The brain has a strictly governed immune response, which relies on the presence of resident cells: microglia and astrocytes. Microglial cells, in particular, are the principal actors of the immune response within the central nervous system [19]. Under physiological conditions, microglia present a quiescent phenotype and act to maintain brain homeostasis through the release of neurotrophic and antiinflammatory factors. Once activated, microglial cells initiate the immune response, stimulating the restoration of tissue homeostasis. Normally, this protective response is concluded after the triggering stress factor has been eliminated. However, if the stimulus is not eradicated, the chronic activation of microglia leads to the establishment of a deleterious permanent inflammatory state that leads to the accumulation of toxic ROS and RNS [19]. The principal ROS/RNS species released by activated microglia are $\mathrm{O}_{2}{ }^{-}$and $\mathrm{NO}^{*}$. The former is produced by nicotinamide adenine dinucleotide phosphate oxidase (NADPH-oxidase), while the latter is synthesized by the nitric oxide synthase (NOS), enzymes that participate to mount the immune response [20]. The importance of microglia dysfunction in PD pathology has been first reported in 1988, when McGeer and colleagues revealed the presence of reactive microglia in SNpc of post-mortem brain tissues from PD patients [21], and the role of neuroinflammation in PD is currently well accepted [22]. Neuromelanin, the dark pigment found in SN dopaminergic cells, has been proposed as an endogenous microglial activator related to PD. Although its role is still controversial, it has been shown that neuromelanin released from dying neurons operates as a microglial trigger, through NADPH-oxidase activation [23]. This phenomenon promotes a feed-forward mechanism that generates a chronic state of inflammation contributing to the further propagation of the neuropathology. As for mitochondria dysfunction, the involvement of neuroinflammation in PD is also supported by the finding that the PD-associated proteins $\alpha$-Synuclein and LRRK2 contribute to or modulate microglia activation [24]. In fact, extracellular $\alpha$-Synuclein aggregates, released by dying neurons, are internalized by microglia, leading to NADPH-oxidase activity and, therefore, to ROS and RNS production [25, 26], while LRRK2 seems to positively regulate the pro-inflammatory response, since its downregulation is associated to a reduced state of inflammation [27, 28]. In conclusion, even though it is still unclear whether neuroinflammation is causative of PD or a consequence of the disorder, its contribution to ROS/RNS production and to the progression of the disease is now well accepted.

\section{Dopamine metabolism}

The underlying cause of the selective dopaminergic cell loss in PD is still an open question. Dopamine itself has been suggested to contribute to the preferential vulnerability of dopaminergic neurons through the generation of oxidative injury $[29,30]$. While dopamine is a stable molecule inside synaptic vesicles, due to the low $\mathrm{pH}$ value, its instability at physiological $\mathrm{pH}$, renders the molecule prone to oxidation at its electron-rich catechol moiety. This leads to the production of hydrogen peroxide and superoxide anion and the formation of highly reactive dopamine-derived quinones (DAQs), which participate in nucleophilic addition reactions [30]. At physiological $\mathrm{pH}$, the cysteine residues of proteins are among the strongest cellular nucleophiles able to interact with DAQs. As these residues are often localized in the active sites of proteins, their interaction with DAQs could be responsible for the alteration of protein functionality, which can result in enzyme inactivation [30]. In addition to proteins, DAQs have been reported to be genotoxic, affecting DNA stability. Their preferential targets are guanine bases, since its N7 position is easily accessible. The formation of DNA adducts is the first step to DNA 
alterations and mutations, which can finally lead to apoptosis [31]. Through its amino moiety, dopamine has been shown to interact with lipid hydroperoxides produced during lipid peroxidation leading to the formation of adducts that could account for the selective cytotoxicity of dopaminergic neurons. Indeed, dopamine adducts derived from polyunsaturated fatty acids are highly reactive and can interfere with normal cellular processes, promoting cytotoxicity. For example, hexanoyl dopamine (HED), an arachidonic acid-derived adduct, has been shown to cause severe cytotoxicity in the human dopaminergic neuroblastoma SH-SY5Y cell line [32].

While PD pathology has been reported to affect also non-dopaminergic cells, particularly during the late stages, and, even though not all dopaminergic neurons are equally affected during PD progression [33], the high toxic potential owned by dopamine itself could contribute to make dopaminergic neurons more sensitive to oxidative damage.

\section{Reactive oxygen species.}

The principal cellular ROS are the superoxide radical $\mathrm{O}_{2}{ }^{-}$ , hydrogen peroxide $\mathrm{H}_{2} \mathrm{O}_{2}$ and hydroxyl radical $\mathrm{OH}^{\circ}$. Among them both $\mathrm{O}_{2}{ }^{*-}$ and $\mathrm{OH}^{*}$ are free radical species, since their orbitals possess one unpaired electron. The superoxide anion $\mathrm{O}_{2}{ }^{-}$is usually referred as the "primary" ROS, because it is capable of interacting with other molecules to form "secondary" ROS [34]. However, $\mathrm{O}_{2}{ }^{-}$ is not highly reactive toward cellular macromolecules such as proteins, nucleic acids and sugars [34]. The superoxide radical has been described to react with proteins, changing their redox state without damaging their structure [35]. Its toxic potential relies principally on the fact the most "secondary" ROS derive from it. Normally, $\mathrm{O}_{2}{ }^{*-}$ is readily detoxified by superoxide dismutases (SODs), which, in turn, release $\mathrm{H}_{2} \mathrm{O}_{2}$ and $\mathrm{O}_{2}$. $\mathrm{H}_{2} \mathrm{O}_{2}$ is inert by itself, but can react with $\mathrm{O}_{2}{ }^{*}$ and iron via Haber-Weiss reaction generating the harmful HO'.

\section{$\mathrm{Fe}(\mathrm{II})+\mathrm{H}_{2} \mathrm{O}_{2} \rightarrow \mathrm{Fe}(\mathrm{III})+\mathrm{HO}^{\bullet}+\mathrm{OH}^{-} \quad$ (Haber-Weiss Reaction)}

The hydroxyl radical has, in vivo, a very short halflife of $10^{-9} \mathrm{~s}$, so that it acts near the site of its formation [20]. This radical is currently considered the most reactive one and the prevention of its synthesis represents the principal protective system [36]. Indeed, $\mathrm{HO}^{\circ}$ possesses a high damaging potential, considering that it avidly reacts with DNA, RNA, proteins and lipids, promoting cytotoxicity. For example, the interaction of $\mathrm{HO}^{*}$ with membrane lipids, induces a reaction called hydrogen abstraction, which generates carbon radicals, which can react with molecular oxygen to produce a lipid peroxyl radicals. These species can then interact with other lipids in close proximity, propagating the reaction and inducing lipid peroxidation [37]. These modifications impact on membrane fluidity and permeability, properties required for optimal cell integrity. Lipid peroxidation is further induced by redox active metal ions such as iron, which forms alkoxyl and peroxyl radicals. Specifically, reduced iron promotes alkoxyl radicals, while the oxidized ion induces peroxyl radicals. Both molecules participate then in lipid peroxidation, exacerbating the state of oxidative injury [37].

Another reactive "secondary" ROS, generated under inflammation by the enzymatically-controlled interaction of $\mathrm{H}_{2} \mathrm{O}_{2}$ with the chloride anion, is hypochlorite $(\mathrm{HOCl})$, a ROS molecule that has also been linked to lipid peroxidation [38].

\section{Mitochondrial ROS production}

Inside cells, mitochondria represent the principal source of $\mathrm{O}_{2}{ }^{-}$, with complex I and III being the major sites of its production. The $\mathrm{O}_{2}{ }^{-}$formed at the level of Complex I is released in the matrix, where it is retained because of it charged state [20]. Here it is converted into $\mathrm{H}_{2} \mathrm{O}_{2}$ by the action of SOD2. Complex $\mathrm{I}$ is the starting point for electron flux in the respiratory chain. From NADH, electrons are accepted by flavin mononucleotide (FMN) and flow to seven iron-sulphur centres, finally arriving to co-enzyme Q (CoQ). $\mathrm{O}_{2}{ }^{--}$is formed by interaction of molecular oxygen with reduced FMN and this reaction is favoured by high NADH/NAD ${ }^{+}$ratios in the matrix. Complex I can induce ROS production through an alternative mechanism which depends on reverse electron transport, a phenomenon that induces a reversion of the electron flux and that has been recently linked to oxidative damage in ischemia-reperfusion injury [39]. When complex II utilizes succinate as substrate, under low adenosine diphosphate (ADP) concentrations, the reduced form of quinone can reduce FMN in complex I, generating $\mathrm{O}_{2}{ }^{-*}$. Although the precise site of production is not clear, this process can be inhibited by rotenone, suggesting that $\mathrm{O}_{2}{ }^{-*}$ synthesis occurs at the FMN site [40].

Complex III, whose physiological activity consists of the transfer of electrons from ubiquinol to cytochrome $\mathrm{c}$, is considered the second site of ROS production. The functional units of this complex are composed of two cytochrome $\mathrm{b}$ subunits forming a pocket into which the ubiquinol can diffuse, transferring electrons to cytochrome c. Through this so-called "Q-cycle", complex III contributes to the hydrogen proton gradient required for ATP synthesis. Disruption of Q-cycle, for example through the inhibitor antimycin, drives $\mathrm{O}_{2}{ }^{--}$formation via interaction of oxygen with ubisemiquinone at the $Q_{0}$ site of complex III. Differently from what described for 
complex I, $\mathrm{O}_{2}{ }^{-*}$ released by complex III is reversed both within the matrix and in the intermembrane space. SOD1 in the intermembrane space detoxifies $\mathrm{O}_{2}{ }^{--}$generating $\mathrm{H}_{2} \mathrm{O}_{2}$, which can passively diffuse through the outer mitochondrial membrane reaching the cytosol. However, $\mathrm{O}_{2}{ }^{-}$has also been reported to cross the outer membrane through the voltage-dependent anion channels (VDAC) thus accumulating in the cytosol [41].

It is worth mentioning that mitochondrial ROS production has been mainly estimated using isolated mitochondria under non physiological conditions [40]. For example, Hansford and co-workers determined that mitochondrial $\mathrm{H}_{2} \mathrm{O}_{2}$ production depends on a high reduction of complex $\mathrm{I}$ and a concomitant elevated membrane potential, conditions achievable only under non physiological conditions [42]. Therefore, although frequently defined as the primary sites of ROS production, mitochondrial complex I and III seem to promote significant ROS accumulation only under pathological circumstances, when there are alterations in their activity due to genetic mutations in the complex-forming proteins or upon exposure to inhibitors, like rotenone or MPTP $[40,43]$. Currently less than $0.1 \%$ of oxygen consumed by these organelles is estimated to be converted into $\mathrm{H}_{2} \mathrm{O}_{2}$ under physiological conditions [44]. In the frame of PD, mitochondrial dysfunction has been suggested to enhance ROS synthesis, inducing their accumulation, which, in turn, leads to cytotoxicity. As recently reviewed, the involvement of mitochondria in PD pathogenesis is supported by post-mortem analyses on PD patients and by genetic and toxin-based animal models of PD [30].

\section{Extra-mitochondrial ROS sources}

Besides the role of mitochondria in ROS generation, other sources cooperate to the production of reactive species synthesis. For example, membrane-bound NADHoxidases (NOX) account for conspicuous ROS production. In the central nervous system, NADHoxidases are mainly expressed in microglia, but they are also found in neurons, astrocytes and several neurovascular regions [45]. These multi-subunit enzymes are assembled after stimulation, as a host-defence mechanism. Although the regulation of their catalytic activities remains unclear, their contribution to superoxide production is well established [46]. Several PD related stimuli, such as $\alpha$-Synuclein oligomers, have been reported to trigger NOX stimulation promoting ROS development [47]. The role of NOX in PD is further supported by the observation that knockout mice for the glycosylate protein gp $91^{\text {phox }}$, the heme binding subunit of NOX, present milder dopaminergic degeneration compared to wild-type littermates [48].
Another source of ROS is represented by the protein myeloperoxidase (MPO), one of the most abundant enzymes of the body responsible for the formation of $\mathrm{HClO}$, from $\mathrm{H}_{2} \mathrm{O}_{2}$ and $\mathrm{Cl}^{-}$, to kill pathogens during the respiratory burst. Being a mannosylated protein, MPO can bind to microglia membranes via mannose receptor, potentiating the anti-microbial function of these cells [49]. Although mainly present in the azurophilic granules of neutrophils, MPO has also been detected in activated microglia of PD patients' brain, suggesting a role for MPO in neuroinflammation [50]. Accordingly, a recent study has demonstrated, in vivo, the efficacy of a selective inhibitor of MPO, called AZD3241, when administered to PD patients for 8 weeks. Indeed, through positron emission tomography study, researchers found a reduction in activated microglia, supporting a role of MPO in neuroinflammation associated to PD [51].

The metalloflavoprotein xanthine oxidase represents another route of $\mathrm{O}_{2}{ }^{--}$synthesis, through nucleic acids degradation. The enzyme catalyses the oxidation of hypoxanthine to xanthine and xanthine to uric acid. This two-step reaction requires molecular oxygen, which is eventually reduced to $\mathrm{O}_{2}{ }^{-}$and $\mathrm{H}_{2} \mathrm{O}_{2}$. However, the involvement of xanthine oxidase in neurodegeneration is less understood [52].

Cyclooxygenase and lipoxygenases are key enzymes involved in the conversion of arachidonic acid to a variety of eicosanoids, such as prostaglandins, thromboxane, and leukotrienes, hormone-like molecules that regulate a wide range of physiological process like blood pressure, platelet aggregation, and inflammation [53]. Both cyclooxygenases and lipoxygenases promote the formation of radical species that can react with molecular oxygen, giving rise to peroxyl radicals [54]. Their involvement in the context of PD is supported by the observation that the inhibition of either 5-lipoxygenase or cyclooxygenase isoform 2 confers protection against MPTP-dependent dopaminergic degeneration in mouse SN [55, 56]. Interestingly, dopaminergic neurons constitute the predominant cellular type where cyclooxygenase 2 is expressed [57].

\section{Reactive nitrogen species.}

This class of molecules encompasses nitric oxide radical $\left(\mathrm{NO}^{\circ}\right)$, peroxynitrite $\left(\mathrm{ONOO}^{-}\right)$, nitrogen dioxide radical $\left(\mathrm{NO}_{2}{ }^{\circ}\right)$ [52]. Nitric oxide (NO) is a nitrogen reactive radical characterized by one unpaired electron. It has a half-life of few seconds in aqueous solution, but its halflife increases up to $15 \mathrm{~s}$ under low oxygen concentrations. Due to its uncharged state, nitric oxide can easily diffuse in the cytoplasm as well as permeate biological membranes [20]. Its biosynthesis depends on the nitric oxide synthase enzyme (NOS), which catalyses the 
conversion of L-arginine to nitric oxide and L-citrulline. Flavin adenine dinucleotide (FAD), FMN, NADPH, tetrahydrobiopterin are all essential cofactors and their absence leads to NOS uncoupling, with formation of $\mathrm{O}_{2}{ }^{-}$ rather than $\mathrm{NO}^{\circ}$. There are three NOS isoforms with distinct functions: neuronal NOS and epithelial NOS, which are activated by calcium flux, and inducible NOS, which is calcium independent. Although its synthesis is associated to pathogen defence, its chronic stimulation leads to biological damages. Indeed, during immune responses or under oxidative stress, $\mathrm{NO}^{*}$ can combine with $\mathrm{O}_{2}{ }^{--}$, producing peroxynitrite $\mathrm{ONOO}^{-}$, a highly oxidizing agent, which induces protein nitration [20]. Related to PD, nitrated $\alpha$-Synuclein has been detected in Lewy Bodies [58] and this modification has been reported to reduce $\alpha$-Synuclein membrane affinity [59]. Peroxynitrite is also a potent source of oxyradicals, since it is readily decomposed into $\mathrm{OH}^{\circ}$ and $\mathrm{NO}_{2}{ }^{\circ}$, independently from the presence of transition metals, potentiating oxidative stress [60]. $\mathrm{NO}^{*}$ has a wide range of targets, including mitochondria, proteins, and lipids. At the mitochondrial level, $\mathrm{NO}^{\circ}$ induces complex III inhibition exhibiting an antimycin-like activity [61]. Moreover, $\mathrm{NO}^{*}$ binds iron-sulfur cluster of cytochrome $c$ oxidoreductase, reversibly inhibiting the enzyme [62]. In $\mathrm{PD}$, microglia represent the principal source of $\mathrm{NO}^{*}$, through the inducible form of NOS enzyme. As aforementioned, upon stimulation, microglial cells mount the "respiratory burst" to kill pathogens. However, chronic microglia activation, with the perpetuation of the inflammatory condition, has been proposed to further exacerbate the oxidative injury, making dopaminergic cells more vulnerable to cell death.

Considering ROS and RNS species and their relative reactivity, it has been hypothesized that while "primary" ROS/RNS have weak cytotoxic potential, "secondary" ROS/RNS present more deleterious effects. The best way that cells have to protect themselves against "secondary" ROS/RNS reactivity is through their avoidance, which can be achieved by a strict control of "primary" species. As most if not all the "secondary" ROS/RNS derive from the superoxide radical, a tight control of its levels appears crucial to preserve cellular functionality.

\section{Physiological functions of ROS and RNS: the other side of the coin}

Besides the role of redox active species in cellular injury, growing evidence now supports their function as stressresponsive mediators on cell signalling pathways. Differently from other cellular mechanisms, redox signalling exploits reactive molecules as messengers, which transfer the information through protein modification, rather than by receptor binding. Indeed, at physiological levels, $\mathrm{H}_{2} \mathrm{O}_{2}$ oxidizes the thiolate anion of cysteine residues, changing protein redox-state. Because cysteines are principally localized in protein active sites, this modification plays an important role in enzymatic catalysis, modulating signalling pathways by protein activation or deactivation according to the $\mathrm{H}_{2} \mathrm{O}_{2}$ target. Another important determinant for ROS and RNS activities relies on their compartmentalization. For example, target proteins of $\mathrm{H}_{2} \mathrm{O}_{2}$ as redox signalling mediator should localize near its production site. Indeed, proteins that are physiologically modified by $\mathrm{H}_{2} \mathrm{O}_{2}$ produced from membrane-bound $\mathrm{NADH}$-oxidase are localized near the membrane [63].

$\mathrm{H}_{2} \mathrm{O}_{2}$ has been shown to participate to several cellular signalling cascades, including NF-kB transcription factor, which is a principal mediator of inflammatory responses. Schmidt and co-workers reported that overexpression of catalase, the enzymatic down-regulator of $\mathrm{H}_{2} \mathrm{O}_{2}$, negatively impacts NF-kB activation, while, in contrast, overexpression of SOD1, which stimulates $\mathrm{H}_{2} \mathrm{O}_{2}$ production by $\mathrm{O}_{2}{ }^{*-}$ dismutation, increases NF-kB activation, supporting a direct role of $\mathrm{H}_{2} \mathrm{O}_{2}$ in the inflammation signalling pathway [64]. Also, $\mathrm{O}_{2}{ }^{-}$has been shown to modulate the immune responses. In particular, $\mathrm{O}_{2}{ }^{-}$generated by mitochondria seems to regulate inflammation through inflammasome activation and cytokines release. In fact, Zhou and colleagues demonstrated that mitochondrial dysfunction due to complex I and III inhibition, leads to "nucleotide-binding domain, leucine-rich family and pyrin domain containing 3" (NLRP3) activation, suggesting a role for $\mathrm{O}_{2}{ }^{*-}$ in inflammasome formation. [65] Coherently, Heid and coworkers showed that the use of Mito-TEMPO, a mitochondrial targeted SOD-mimetic, reduced IL-1 $\beta$ secretion after nigericin or ATP exposure. IL- $1 \beta$ secretion is an early event in inflammasome assembling [66]. Reactive species have also been described to modulate gene expression. The nuclear transcription factorerythroid 2-related factor 2 (Nrf2) is the master transcription factor for anti-oxidant responses and it drives the expression of more than 100 genes involved in cellular protection. Under basal conditions, Nrf2 is inhibited by kelch-like ECH-associated protein (Keap1), which destabilizes Nrf2, promoting its degradation. Importantly, Keap1 possesses highly reactive cysteine residues, which can be oxidized upon exposure to oxidative stimuli, removing Keap1 inhibitory activity. Hence, under oxidative conditions, through the action of redox mediators, Nrf2is transferred to the nucleus where it stimulates the expression of cytoprotective genes [67]. 
Table 1. Physiological functions ascribed to ROS and RNS.

\begin{tabular}{llr}
\hline ROS/RNS species & Physiological functions & Reference \\
\hline $\mathbf{O}_{2}{ }^{--}$ & Inflammasome activation and cytokines release & {$[65,66]$} \\
& $\begin{array}{l}\text { Modification of protein activity. Modulation of signalling pathways (NF-kB) } \\
\text { and gene expression (Nrf2) }\end{array}$ & {$[64,67]$} \\
$\mathbf{H}_{2} \mathbf{O}_{2}$ & $\begin{array}{l}\text { Vasodilatation, platelet aggregation, neuronal firing, synaptic plasticity, and } \\
\text { nourotransmitter release }\end{array}$ & {$[68,69]$} \\
\hline
\end{tabular}

Not only ROS but also RNS have fundamental physiological functions. For example, $\mathrm{NO}^{\circ}$ participates to regulate vasodilatation, platelet aggregation, neuronal firing, synaptic plasticity, and neurotransmitter release [68]. Nitric oxide is considered an atypical neurotransmitter, since it is not stored in synaptic vesicles and it does not bind to receptors. Its synthesis is induced after stimulation of N-methyl-D-aspartate (NMDA) glutamate receptors and it is released immediately, diffusing through the pre-synaptic membrane. The principal molecular activity is the induction of guanylyl cyclase enzyme that synthesizes guanosine 3 ' 5 '-cyclic monophosphate (cGMP) a second messenger involved in multiple signalling pathways. Interestingly, it has been reported that $\mathrm{NO}^{\circ}$, via cGMP, also mediates long-term potentiation and long-term depression, two mechanisms involved in learning and memory processes [69]. Table 1 summarizes the main signalling functions ascribed to ROS and RNS.

These data support a role for ROS and RNS in signalling cascades, evidencing a protective function, exerted under physiological concentrations and in specific cellular compartments. Indeed, ROS and RNS are now considered dual-faceted molecules, which not only act as damaging agents, due to their chemical nature, but also positively contribute to cell homeostasis. What differentiates ROS and RNS as signalling molecules or damaging agents depends on their concentrations. [63]. What is not still fully understood is whether all ROS/RNS species are involved in cell signalling. Many studies support the physiological function of $\mathrm{O}_{2}{ }^{-}, \mathrm{H}_{2} \mathrm{O}_{2}$, and $\mathrm{NO}^{\circ}$ (Table 1), while for other "secondary" species, only detrimental actions are reported. In conclusion, the cellular effects of a reactive species should be measured considering its relative concentration and cellular location and the mechanisms involved in its production and clearance.

\section{Antioxidants in PD therapy}

PD is still an incurable disorder. While the available medications only treat the symptoms of $\mathrm{PD}$, drugs that can arrest, reduce and/or delay the death of dopaminergic neurons and reverse the effects of the disease are not yet available. The current pharmacological therapy to replace the loss of dopamine is mainly based on the administration of L-DOPA, a dopamine precursor that crosses the bloodbrain barrier (BBB). Unfortunately, L-DOPA therapy often loses its efficacy after few years of treatment, emphasising the urgent need for additional pharmacological approaches. As it is clear from what is discussed above, oxidative damage participates in neuron degeneration in PD. It follows that antioxidants should represent a valuable therapeutic approach to cope with the disease progression. Nevertheless, as recently reviewed [70-72], the effects attained in most of the controlled clinical trials are rather disappointing with very modest results. Several reasons could account for these disappointing clinical outcomes and their knowledge could provide the rationale to design novel strategies.

First, a good therapeutic candidate must be able to survive ingestion, cross the intestinal barrier and reach the blood circulation without being degraded too rapidly in the plasma or eliminated through the kidneys. Moreover, it must be able to pass through cell membranes and, even more importantly, to cross the BBB. So, it is possible that the antioxidants used until now in clinical trials were not effective in reducing ROS/RNS levels because the required effective concentration was never reached on the target. Unfortunately, this issue cannot be easily addressed in vivo because of technical problems in measuring ROS concentrations and their variations [73]. Plasma levels of the supplemented antioxidants can be more easily monitored, even though this information can neither be easily related to the drug levels in the brain and, in particular, in dopaminergic neurons, nor to the effective elimination of ROS/RNS. Moreover, subsequently to their 
assumption, antioxidants could evenly distribute inside the body while the oxidative injury is mainly restricted to a specific region such as dopaminergic neurons in SNpc. As a consequence, the local concentration of antioxidants in the region of interest could be inadequate to deal with specific and local needs. Another point that must be considered is that, in addition to being harmful when they are present in excess, ROS/RNS play also important physiological functions, mainly as signalling mediators. As previously described, it is now evident that they affect several cellular functions such as proliferation, metabolism, differentiation, and survival, participate to the anti-oxidant and anti-inflammatory response and to iron metabolism [74]. It follows that their substantial removal can result in toxic rather than protective effects and a valuable therapeutic strategy should be aimed at keeping oxidant species at around the functional concentration rather than try to totally remove them.

In addition to the abovementioned explanations, focused on quantitative aspects, a central issue concerning most of the antioxidant-based clinical trials is that the real nature of the radical species actually involved in the progression of PD has never been carefully considered. As considered in the first part of this review, when discussing about ROS/RNS and their scavengers, it is crucial to know which kind of ROS/RNS is involved, because their chemical reactivity is different as well as their cellular targets. In particular, while "secondary" ROS/RNS have been demonstrated to be much more reactive than the "primary" ones, it is also clear that most if not all of them derive from the superoxide radical. In this frame, a key pitfall of the previously tested antioxidant therapies may be that they did not specifically target what could represent the primary cause of oxidative stress, i.e. excessive superoxide anion production, but rather the downstream effects (production of hydrogen peroxide, hydroxyl radical, peroxynitrite...). This is likely to be the case, for example, of $\alpha$-tocopherol (vitamin E), ascorbic acid (vitamin C), creatine and apocynin [75]. In our opinion, a treatment strategy for oxidative stress is likely to be more effective if it targets the origin of ROS generation. This issue could be tackled by the administration of a mixture of antioxidant molecules directed towards different ROS species with the potential caveat that also side effects may act in a synergistic way. Alternatively, superoxide anions might be a potential therapeutic target for new drugs. In other words, antioxidant molecules, capable of decreasing superoxide radicals, could represent a potential therapeutic strategy to counteract the progression of PD.

\section{Superoxide radical dismutation and therapeutic implications}

For their ability to convert superoxide radicals to molecular oxygen and hydrogen peroxide, Superoxide dismutase enzymes (SODs) are usually considered the first line of defence against reactive oxygen species. Two different SOD isoenzymes, contribute to superoxide anions removal inside cells. SOD1 is a copper/zinc protein located in the cytosol and in the mitochondrial intermembrane space, but also present in peroxisomes and in the nucleus. SOD2 is a mitochondrial manganese enzyme, which is the main scavenger of superoxide anions produced during the mitochondrial oxidative phosphorylation. Interestingly, both SOD1 and SOD2 transgenic mice have been demonstrated to be resistant to MPTP-induced neurotoxicity, providing evidence that some of the deleterious effects of MPTP could be mediated by superoxide radicals and strongly supporting the possibility that these radical species could play a significant role in the aetiology of PD [76, 77].

In light of these considerations, we recently evaluated the beneficial role of SODs against paraquatinduced toxicity in human neuroblastoma SH-SY5Y cell line and in Drosophila melanogaster [78]. Epidemiological studies demonstrated that chronic exposure to pesticides, such as PQ and rotenone, is associated with a higher risk of developing PD [79, 80]. Consistently, two independent meta-analyses found an association between pesticides, in particular PQ, and the risk of PD [81, 82]. The picture that emerges from our study emphasizes the role of both cytosolic and mitochondrial SODs in protecting cells against superoxide overproduction. Specifically, while in cells or flies treated with high concentrations of PQ (acute treatment), we observed a critical role played by SOD2 in protecting against oxidative damage, the situation observed in flies at sub-lethal concentrations of PQ (chronic treatment) indicates that only the overexpression of SOD1 is able to rescue the PQ-associated toxicity, while SOD2 appears ineffective. Interestingly, this is also true when SOD1 is specifically expressed in dopaminergic neurons [78]. Our results are in agreement with the notion that dopaminergic neurons are particularly vulnerable to oxidative conditions, and that other cytosolic processes inside these neurons, such as dopamine oxidation, may amplify the toxicity derived from an elevated production of free radical species.

Although SODs cannot be directly exploited from a therapeutic point of view, primarily for their inability to cross the $\mathrm{BBB}$, still the protective effects observed with both SOD1 and SOD2 emphasize the possibility to explore the therapeutic potential of SOD mimetic compounds, i.e., small molecules possessing SOD-like catalytic properties. Four main classes of molecules possessing SOD-like activity have been described, which include metalloporphyrin, nitroxides, $\mathrm{Mn}(\mathrm{III})$-salen 
complexes and $\mathrm{Mn}(\mathrm{II})$-cyclic polyamines [83, 84]. Among them, Mn-porphyrins and Mn(II)-cyclic polyamines appear the most interesting from a chemical and therapeutic standpoint.
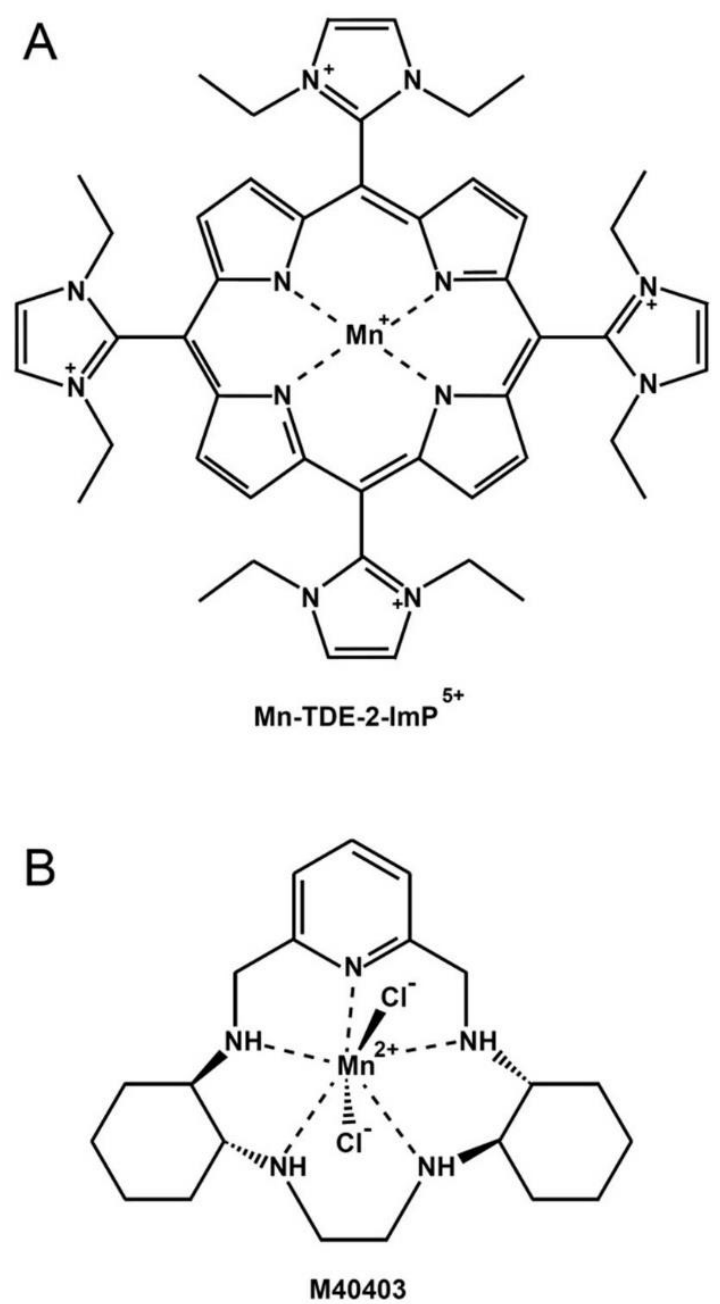

Figure 2. SOD-mimetic compounds. (A) MnTDE-2-ImP ${ }^{5+}$, a Mn-porphyrin and (B) M40403, a Mn(II)-cyclic polyamine are among the most interesting SOD-mimetic molecules from a therapeutic standpoint.

\section{Porphyrin-based SOD mimetics}

Porphyrin-based SOD mimetics include more than 50 molecules, which differ in their chemico-physical and functional characteristics. In particular, over the years many studies have sought to obtain water-soluble molecules with high catalytic activities, low toxicity [84, 85]. It is now clear that Mn-porphyrins are not simple
SOD-mimetics because they also possess $\mathrm{ONOO}^{-}$ scavenger capability and they are able to modulate redoxsensitive cellular transcriptional activity [84]. For the reasons discussed above, the lack of specificity could represent a limit for the utilization of such molecules in clinical trials. Another possible drawback arises from a pro-oxidative activity attributed to Mn-porphyrins in the presence of cellular reductants [84]. While this feature can be therapeutically exploited in some circumstances, for example to fight cancer [86], it might make the use of Mnporphyrins in PD more difficult. A big effort to develop new Mn-porphyrins toward clinical trial is currently being carried out and some molecules are already under clinical trials. Among them, the compound called MnTDE-2$\mathrm{ImP}^{5+}$ (AEOL 10150) (Fig. 2A) resulted safe and well tolerated in a Phase I clinical trial on Amyotrophic Lateral Sclerosis patients and is now pursued as a radioprotector by the pharmaceutical company Aeolus Pharmaceuticals [87]. More importantly in the context of PD, the same company is currently working on optimization of manufacturing process and formulation of another Mnporphyrin, AEOL 11114, which shows promise in PD, being protective in a MPTP-based mouse model of PD [88]. Very interestingly, the molecule has been demonstrated to be orally active, to have relatively long plasma half-life, rapid absorption by the gastrointestinal tract, ability to penetrate the $\mathrm{BBB}$, and can achieve therapeutically active concentrations in the brain [88].

\section{Mn(II)-cyclic polyamines-based SOD mimetics}

At the end of the last century, a new class of low molecular mass, Mn(II)-pentaazamacrocyclic ligandbased SOD mimetics has been developed to protect against inflammation in those disease-related conditions in which native SOD enzymes were found effective in animal models. This class is exemplified by the prototypical complex M40403 (Fig. 2B), a derivative of the 15-membered macrocyclic ligand 1,4,7,10,13pentaazacyclopentadecane that contains added bis(cyclohexylpyridine) functionalities. The molecule was first tested in rat models of inflammation and ischemia-reperfusion injury showing high protection [89]. M40403 is a stable $\mathrm{Mn}$ (II) complex, which is resistant to oxidative degradation and is excreted intact with no detectable dissociation in vivo. Its catalytic rate constant has been demonstrated to be $\mathrm{pH}$ dependent with a rate at pH 6.0 exceeding $2 \times 10^{8} \mathrm{M}^{-1} \mathrm{~s}^{-1}$ (comparable to the native SOD2 enzymatic activity, while at $\mathrm{pH} 7.4$ the rate is an order of magnitude lower $\left(2 \times 10^{7} \mathrm{M}^{-1} \mathrm{~s}^{-1}\right)$ [89]. Moreover, the molecule is water-soluble so that it can be orally administered and it is able to cross the BBB [89]. An important characteristic of M40403 is its ability to catalytically remove superoxide radicals in a very 
selective manner being that it does not react with other biologically relevant oxidizing species, such as, hydrogen peroxide, hypochlorite, nitric oxide and peroxynitrite [90]. Although other $\mathrm{Mn}$ (II)-cyclic polyamines-based SOD mimetics similar to M40403 were synthesized aimed at improving the catalytic activity while preserving its stability, until very recently only M40403 was tested in phase I and II clinical trials for the treatment of pain in approximately 700 subjects/patients, resulting in being safe and well-tolerated [91]. The pharmaceutical company Galera Therapeutic is currently pursuing the development of such a class of SOD mimetics to offer novel therapeutics to improve both the tolerability of current anti-cancer treatments and directly treat cancer. They are currently recruiting participant for a randomized Phase II clinical trial to assess the effect of their lead clinical candidate GC4419 on the incidence, severity and duration of severe oral mucositis when given to patients with squamous cell cancers of the head and neck in combination with radiation and chemotherapy (NCT02508389).

Given the positive results we obtained in our PQbased cellular and fly models of PD and considering the aforementioned properties of M40403, we analysed its ability to prevent the PQ-induced toxicity. Not only M40403 showed significant beneficial properties in our cellular model, but, when tested in vivo, it succeeded in rescuing the lethality induced by elevated concentrations of PQ. Moreover, in the presence of a sub-lethal concentration of $\mathrm{PQ}, \mathrm{M} 40403$ was also able to improve the locomotion behaviour of flies, a prominent aspect in the context of PD whose main clinical hallmark is represented by motor dysfunction. The protection observed with M40403, both in the presence of acute and chronic PQ treatments, is probably due to its ability to act in vivo both at cytosolic and mitochondrial level. Accordingly, we demonstrated that the molecule is able to rescue the toxicity induced by the down-regulation of either SOD1 or SOD2, indicating that it can act both at cytosolic and mitochondrial level [78]. This characteristic is of particular importance considering that in PD both cytosolic and mitochondrial processes could contribute to and exacerbate the production of superoxide radicals.

\section{Conclusions}

In spite of the disappointing results obtained in the antioxidant-based clinical trials carried out until now to hamper the progression of PD, the use of antioxidant compounds could still be considered for their therapeutic potential, providing that the mechanisms underlying the oxidative injury associated to the disease are better understood. This knowledge is critical to design effective molecules able to selectively reduce the levels of defined damaging reactive species rather than eliminate ROS/RNS in a nonspecific way. A key drawback of the previously tested antioxidant therapies designed to cope with PD is that they did not specifically target what could represent the primary cause of oxidative stress, but rather the downstream effects. As several indications now exist highlighting the prominent role of the superoxide radical in inducing neuronal toxicity, a new therapeutic opportunity, to be carefully evaluated, is the use of SODmimetic molecules, which can selectively remove superoxide radicals in a catalytic way. The latter is a key aspect in the future prospective of these molecules: the challenge of only eliminating the ROS associated to the induce damages without interfering with the signalling activity can be address by modulating the dose going down from a proved effective concentration. Many SODmimetics are currently available, even though only few of them seem to have therapeutically exploitable properties. As these compounds have been initially designed to protect against inflammatory processes, a further advantage of their use is that, besides reducing oxidative damages inside cells, they might have the potential to counteract microglia chronic activation, one of the proposed cause of disease progression in PD. Nevertheless, caution must be applied when considering the protective properties of such molecules. Although porphyrin- and $\mathrm{Mn}$ (II)-cyclic polyamines-based SOD mimetics have shown very promising features that might be exploited to cope with PD, the experimental data describing their protective effects are mainly derived from toxin-based animal models of the disease. Unfortunately, antioxidants having potential therapeutic properties in animal models of PD very often showed disappointing results when tested in clinical trials. The main concern in this regard is that, even though a multiplicity of PDrelated models exists, none of them fully recapitulate the clinical and behavioural phenotypes associated with the disease. For example, the MPTP-based mouse model has been the most used in pre-clinical studies, but the encouraging results obtained in this model were almost never reproduced in clinical trials, indicating that it is far from being a perfect model. It follows that, before initiating a new clinical study, to increase the probability of its success, different approaches should be explored, taking into consideration several experimental paradigms with the use, for example, of animal models related to both sporadic and familial forms of PD. In conclusion, with the necessary cautions, superoxide radical dismutation might represent a new promising therapeutic target in Parkinson's disease that should be worthy of further consideration. 


\section{Acknowledgments}

This work was supported by a grant from the Italian Ministry of Education, University and Research (2015T778JW).

\section{References}

[1] Kalia LV, Lang AE (2015). Parkinson's disease. Lancet, 386: 896-912

[2] Dickson DW (2012). Parkinson's disease and parkinsonism: neuropathology. Cold Spring Harb Perspect Med, 2

[3] Blesa J, Trigo-Damas I, Quiroga-Varela A, JacksonLewis VR (2015). Oxidative stress and Parkinson's disease. Front Neuroanat, 9: 91

[4] Jiang T, Sun Q, Chen S (2016). Oxidative stress: A major pathogenesis and potential therapeutic target of antioxidative agents in Parkinson's disease and Alzheimer's disease. Prog Neurobiol, 147: 1-19

[5] Dexter DT, Jenner P (2013). Parkinson disease: from pathology to molecular disease mechanisms. Free Radic Biol Med, 62: 132-144

[6] Dias V, Junn E, Mouradian MM (2013). The role of oxidative stress in Parkinson's disease. J Parkinsons Dis, 3: 461-491

[7] Cadenas E, Davies KJ (2000). Mitochondrial free radical generation, oxidative stress, and aging. Free Radic Biol Med, 29: 222-230

[8] Langston JW, Ballard P, Tetrud JW, Irwin I (1983). Chronic Parkinsonism in humans due to a product of meperidine-analog synthesis. Science, 219: 979-980

[9] Bose A, Beal MF (2016). Mitochondrial dysfunction in Parkinson's disease. J Neurochem, 139 Suppl 1: 216-231

[10] Schapira AH, Mann VM, Cooper JM, Dexter D, Daniel SE, Jenner P, et al. (1990). Anatomic and disease specificity of NADH CoQ1 reductase (complex I) deficiency in Parkinson's disease. J Neurochem, 55: 2142-2145

[11] Hattori N, Tanaka M, Ozawa T, Mizuno Y (1991). Immunohistochemical studies on complexes I, II, III, and IV of mitochondria in Parkinson's disease. Ann Neurol, 30: $563-571$

[12] Hattingen E, Magerkurth J, Pilatus U, Mozer A, Seifried C, Steinmetz H, et al. (2009). Phosphorus and proton magnetic resonance spectroscopy demonstrates mitochondrial dysfunction in early and advanced Parkinson's disease. Brain, 132: 3285-3297

[13] Yang Y, Gehrke S, Imai Y, Huang Z, Ouyang Y, Wang JW, et al. (2006). Mitochondrial pathology and muscle and dopaminergic neuron degeneration caused by inactivation of Drosophila Pink1 is rescued by Parkin. Proc Natl Acad Sci U S A, 103: 10793-10798

[14] Park J, Lee SB, Lee S, Kim Y, Song S, Kim S, et al. (2006). Mitochondrial dysfunction in Drosophila PINK1 mutants is complemented by parkin. Nature, 441: 11571161

[15] Greene JC, Whitworth AJ, Kuo I, Andrews LA, Feany MB, Pallanck LJ (2003). Mitochondrial pathology and apoptotic muscle degeneration in Drosophila parkin mutants. Proc Natl Acad Sci U S A, 100: 4078-4083

[16] Wang X, Petrie TG, Liu Y, Liu J, Fujioka H, Zhu X (2012). Parkinson's disease-associated DJ-1 mutations impair mitochondrial dynamics and cause mitochondrial dysfunction. J Neurochem, 121: 830-839

[17] Junn E, Jang WH, Zhao X, Jeong BS, Mouradian MM (2009). Mitochondrial localization of DJ-1 leads to enhanced neuroprotection. J Neurosci Res, 87: 123-129

[18] Hayashi T, Ishimori C, Takahashi-Niki K, Taira T, Kim YC, Maita H, et al. (2009). DJ-1 binds to mitochondrial complex I and maintains its activity. Biochem Biophys Res Commun, 390: 667-672

[19] Kettenmann H, Hanisch UK, Noda M, Verkhratsky A (2011). Physiology of microglia. Physiol Rev, 91: 461553

[20] Valko M, Leibfritz D, Moncol J, Cronin MT, Mazur M, Telser J (2007). Free radicals and antioxidants in normal physiological functions and human disease. Int $\mathbf{J}$ Biochem Cell Biol, 39: 44-84

[21] McGeer PL, Itagaki S, Boyes BE, McGeer EG (1988). Reactive microglia are positive for HLA-DR in the substantia nigra of Parkinson's and Alzheimer's disease brains. Neurology, 38: 1285-1291

[22] Greggio E, Civiero L, Bisaglia M, Bubacco L (2012). Parkinson's disease and immune system: is the culprit LRRKing in the periphery? J Neuroinflammation, 9: 94

[23] Zhang W, Phillips K, Wielgus AR, Liu J, Albertini A, Zucca FA, et al. (2011). Neuromelanin activates microglia and induces degeneration of dopaminergic neurons: implications for progression of Parkinson's disease. Neurotox Res, 19: 63-72

[24] Schapansky J, Nardozzi JD, LaVoie MJ (2015). The complex relationships between microglia, alphasynuclein, and LRRK2 in Parkinson's disease. Neuroscience, 302: 74-88

[25] Zhang W, Wang T, Pei Z, Miller DS, Wu X, Block ML, et al. (2005). Aggregated alpha-synuclein activates microglia: a process leading to disease progression in Parkinson's disease. FASEB J, 19: 533-542

[26] Gao HM, Zhang F, Zhou H, Kam W, Wilson B, Hong JS (2011). Neuroinflammation and alpha-synuclein dysfunction potentiate each other, driving chronic progression of neurodegeneration in a mouse model of Parkinson's disease. Environ Health Perspect, 119: 807814

[27] Russo I, Berti G, Plotegher N, Bernardo G, Filograna R, Bubacco L, et al. (2015). Leucine-rich repeat kinase 2 positively regulates inflammation and down-regulates NF-kappaB p50 signaling in cultured microglia cells. J Neuroinflammation, 12: 230

[28] Moehle MS, Webber PJ, Tse T, Sukar N, Standaert DG, DeSilva TM, et al. (2012). LRRK2 inhibition attenuates microglial inflammatory responses. J Neurosci, 32: 1602-1611

[29] Bisaglia M, Greggio E, Beltramini M, Bubacco L (2013). Dysfunction of dopamine homeostasis: clues in the hunt for novel Parkinson's disease therapies. FASEB J, 27: 2101-2110 
[30] Bisaglia M, Filograna R, Beltramini M, Bubacco L (2014). Are dopamine derivatives implicated in the pathogenesis of Parkinson's disease? Ageing Res Rev, 13: $107-114$

[31] Stokes AH, Hastings TG, Vrana KE (1999). Cytotoxic and genotoxic potential of dopamine. J Neurosci Res, 55: 659-665

[32] Liu X, Yamada N, Maruyama W, Osawa T (2008). Formation of dopamine adducts derived from brain polyunsaturated fatty acids: mechanism for Parkinson disease. J Biol Chem, 283: 34887-34895

[33] Surmeier DJ, Guzman JN, Sanchez-Padilla J, Goldberg JA (2010). What causes the death of dopaminergic neurons in Parkinson's disease? Prog Brain Res, 183: 5977

[34] Valko M, Rhodes CJ, Moncol J, Izakovic M, Mazur M (2006). Free radicals, metals and antioxidants in oxidative stress-induced cancer. Chem Biol Interact, 160: $1-40$

[35] Weidinger A, Kozlov AV (2015). Biological Activities of Reactive Oxygen and Nitrogen Species: Oxidative Stress versus Signal Transduction. Biomolecules, 5: 472484

[36] Lushchak VI (2014). Free radicals, reactive oxygen species, oxidative stress and its classification. Chem Biol Interact, 224: 164-175

[37] Halliwell B, Gutteridge JM (1984). Oxygen toxicity, oxygen radicals, transition metals and disease. Biochem J, 219: 1-14

[38] Spickett CM, Jerlich A, Panasenko OM, Arnhold J, Pitt AR, Stelmaszynska T, et al. (2000). The reactions of hypochlorous acid, the reactive oxygen species produced by myeloperoxidase, with lipids. Acta Biochim Pol, 47: 889-899

[39] Chouchani ET, Pell VR, James AM, Work LM, SaebParsy K, Frezza C, et al. (2016). A Unifying Mechanism for Mitochondrial Superoxide Production during Ischemia-Reperfusion Injury. Cell Metab, 23: 254-263

[40] Murphy MP (2009). How mitochondria produce reactive oxygen species. Biochem J, 417: 1-13

[41] Han D, Antunes F, Canali R, Rettori D, Cadenas E (2003). Voltage-dependent anion channels control the release of the superoxide anion from mitochondria to cytosol. J Biol Chem, 278: 5557-5563

[42] Hansford RG, Hogue BA, Mildaziene V (1997). Dependence of $\mathrm{H} 2 \mathrm{O} 2$ formation by rat heart mitochondria on substrate availability and donor age. J Bioenerg Biomembr, 29: 89-95

[43] Brand MD (2010). The sites and topology of mitochondrial superoxide production. Exp Gerontol, 45: 466-472

[44] Alexeyev MF (2009). Is there more to aging than mitochondrial DNA and reactive oxygen species? FEBS J, 276: 5768-5787

[45] Nayernia Z, Jaquet V, Krause KH (2014). New insights on NOX enzymes in the central nervous system. Antioxid Redox Signal, 20: 2815-2837

[46] Hernandes MS, Britto LR (2012). NADPH oxidase and neurodegeneration. Curr Neuropharmacol, 10: 321-327
[47] Jiang T, Hoekstra J, Heng X, Kang W, Ding J, Liu J, et al. (2015). P2X7 receptor is critical in alpha-synuclein-mediated microglial NADPH oxidase activation. Neurobiol Aging, 36: 2304-2318

[48] Zhang W, Wang T, Qin L, Gao HM, Wilson B, Ali SF, et al. (2004). Neuroprotective effect of dextromethorphan in the MPTP Parkinson's disease model: role of NADPH oxidase. FASEB J, 18: 589-591

[49] Lefkowitz DL, Lefkowitz SS (2008). Microglia and myeloperoxidase: a deadly partnership in neurodegenerative disease. Free Radic Biol Med, 45: 726-731

[50] Gellhaar S, Sunnemark D, Eriksson H, Olson L, Galter D (2017). Myeloperoxidase-immunoreactive cells are significantly increased in brain areas affected by neurodegeneration in Parkinson's and Alzheimer's disease. Cell Tissue Res,

[51] Jucaite A, Svenningsson P, Rinne JO, Cselenyi Z, Varnas K, Johnstrom P, et al. (2015). Effect of the myeloperoxidase inhibitor AZD3241 on microglia: a PET study in Parkinson's disease. Brain, 138: 2687-2700

[52] Dhawan V (2014) Reactive Oxygen and Nitrogen Species: General Considerations. In Studies on Respiratory Disorders (Ganguly, N. K., Jindal, S. K., Biswal, S., Barnes, P. J., Pawankar, R., eds) pp. 27-47, Springer New York, New York, NY

[53] Dennis EA, Norris PC (2015). Eicosanoid storm in infection and inflammation. Nat Rev Immunol, 15: 511523

[54] Schneider C, Pratt DA, Porter NA, Brash AR (2007). Control of oxygenation in lipoxygenase and cyclooxygenase catalysis. Chem Biol, 14: 473-488

[55] Kang KH, Liou HH, Hour MJ, Liou HC, Fu WM (2013). Protection of dopaminergic neurons by 5-lipoxygenase inhibitor. Neuropharmacology, 73: 380-387

[56] Feng ZH, Wang TG, Li DD, Fung P, Wilson BC, Liu B, et al. (2002). Cyclooxygenase-2-deficient mice are resistant to 1-methyl-4-phenyl1, 2, 3, 6tetrahydropyridine-induced damage of dopaminergic neurons in the substantia nigra. Neurosci Lett, 329: 354358

[57] Teismann P (2012). COX-2 in the neurodegenerative process of Parkinson's disease. Biofactors, 38: 395-397

[58] Giasson BI, Duda JE, Murray IV, Chen Q, Souza JM, Hurtig HI, et al. (2000). Oxidative damage linked to neurodegeneration by selective alpha-synuclein nitration in synucleinopathy lesions. Science, 290: 985-989

[59] Hodara R, Norris EH, Giasson BI, Mishizen-Eberz AJ, Lynch DR, Lee VM, et al. (2004). Functional consequences of alpha-synuclein tyrosine nitration: diminished binding to lipid vesicles and increased fibril formation. J Biol Chem, 279: 47746-47753

[60] Pisoschi AM, Pop A (2015). The role of antioxidants in the chemistry of oxidative stress: A review. Eur J Med Chem, 97: 55-74

[61] Iglesias DE, Bombicino SS, Valdez LB, Boveris A (2015). Nitric oxide interacts with mitochondrial complex III producing antimycin-like effects. Free Radic Biol Med, 89: 602-613 
[62] Cleeter MW, Cooper JM, Darley-Usmar VM, Moncada S, Schapira AH (1994). Reversible inhibition of cytochrome c oxidase, the terminal enzyme of the mitochondrial respiratory chain, by nitric oxide. Implications for neurodegenerative diseases. FEBS Lett, 345: 50-54

[63] Schieber M, Chandel NS (2014). ROS function in redox signaling and oxidative stress. Curr Biol, 24: R453-462

[64] Schmidt KN, Amstad P, Cerutti P, Baeuerle PA (1995). The roles of hydrogen peroxide and superoxide as messengers in the activation of transcription factor NFkappa B. Chem Biol, 2: 13-22

[65] Zhou R, Yazdi AS, Menu P, Tschopp J (2011). A role for mitochondria in NLRP3 inflammasome activation. Nature, 469: 221-225

[66] Heid ME, Keyel PA, Kamga C, Shiva S, Watkins SC, Salter RD (2013). Mitochondrial reactive oxygen species induces NLRP3-dependent lysosomal damage and inflammasome activation. J Immunol, 191: 5230-5238

[67] Ma Q (2013). Role of nrf2 in oxidative stress and toxicity. Annu Rev Pharmacol Toxicol, 53: 401-426

[68] Jimenez-Jimenez FJ, Alonso-Navarro H, Herrero MT, Garcia-Martin E, Agundez JA (2016). An Update on the Role of Nitric Oxide in the Neurodegenerative Processes of Parkinson's Disease. Curr Med Chem, 23: 2666-2679

[69] Paul V, Ekambaram P (2011). Involvement of nitric oxide in learning \& memory processes. Indian $\mathrm{J}$ Med Res, 133: 471-478

[70] Murphy MP (2014). Antioxidants as therapies: can we improve on nature? Free Radic Biol Med, 66: 20-23

[71] Schmidt HH, Stocker R, Vollbracht C, Paulsen G, Riley D, Daiber A, et al. (2015). Antioxidants in Translational Medicine. Antioxid Redox Signal, 23: 1130-1143

[72] Filograna R, Beltramini M, Bubacco L, Bisaglia M (2016). Anti-Oxidants in Parkinson's Disease Therapy: A Critical Point of View. Curr Neuropharmacol, 14: 260271

[73] Sanz A (2016). Mitochondrial reactive oxygen species: Do they extend or shorten animal lifespan? Biochim Biophys Acta, 1857: 1116-1126

[74] Ray PD, Huang BW, Tsuji Y (2012). Reactive oxygen species (ROS) homeostasis and redox regulation in cellular signaling. Cell Signal, 24: 981-990

[75] Jin H, Kanthasamy A, Ghosh A, Anantharam V, Kalyanaraman B, Kanthasamy AG (2014). Mitochondria-targeted antioxidants for treatment of Parkinson's disease: preclinical and clinical outcomes. Biochim Biophys Acta, 1842: 1282-1294

[76] Przedborski S, Kostic V, Jackson-Lewis V, Naini AB, Simonetti S, Fahn S, et al. (1992). Transgenic mice with increased $\mathrm{Cu} / \mathrm{Zn}$-superoxide dismutase activity are resistant to N-methyl-4-phenyl-1,2,3,6tetrahydropyridine-induced neurotoxicity. J Neurosci, 12: 1658-1667

[77] Klivenyi P, St Clair D, Wermer M, Yen HC, Oberley T, Yang L, et al. (1998). Manganese superoxide dismutase overexpression attenuates MPTP toxicity. Neurobiol Dis, 5: 253-258
[78] Filograna R, Godena VK, Sanchez-Martinez A, Ferrari E, Casella L, Beltramini M, et al. (2016). Superoxide Dismutase (SOD)-mimetic M40403 Is Protective in Cell and Fly Models of Paraquat Toxicity: IMPLICATIONS FOR PARKINSON DISEASE. J Biol Chem, 291: 92579267

[79] Tanner CM, Kamel F, Ross GW, Hoppin JA, Goldman SM, Korell M, et al. (2011). Rotenone, paraquat, and Parkinson's disease. Environ Health Perspect, 119: 866872

[80] Wang A, Costello S, Cockburn M, Zhang X, Bronstein J, Ritz B (2011). Parkinson's disease risk from ambient exposure to pesticides. Eur J Epidemiol, 26: 547-555

[81] van der Mark M, Brouwer M, Kromhout H, Nijssen P, Huss A, Vermeulen R (2012). Is pesticide use related to Parkinson disease? Some clues to heterogeneity in study results. Environ Health Perspect, 120: 340-347

[82] Pezzoli G, Cereda E (2013). Exposure to pesticides or solvents and risk of Parkinson disease. Neurology, 80: 2035-2041

[83] Salvemini D, Mazzon E, Dugo L, Riley DP, Serraino I, Caputi AP, et al. (2001). Pharmacological manipulation of the inflammatory cascade by the superoxide dismutase mimetic, M40403. Br J Pharmacol, 132: 815-827

[84] Batinic-Haberle I, Reboucas JS, Spasojevic I (2010). Superoxide dismutase mimics: chemistry, pharmacology, and therapeutic potential. Antioxid Redox Signal, 13: 877-918

[85] Rajic Z, Tovmasyan A, de Santana OL, Peixoto IN, Spasojevic I, do Monte SA, et al. (2017). Challenges encountered during development of Mn porphyrinbased, potent redox-active drug and superoxide dismutase mimic, MnTnBuOE-2-PyP5+, and its alkoxyalkyl analogues. J Inorg Biochem, 169: 50-60

[86] Tovmasyan A, Sampaio RS, Boss MK, Bueno-Janice JC, Bader BH, Thomas M, et al. (2015). Anticancer therapeutic potential of Mn porphyrin/ascorbate system. Free Radic Biol Med, 89: 1231-1247

[87] Batinic-Haberle I, Tovmasyan A, Spasojevic I (2016). Opinion on Schmidt et al. Antioxid Redox Signal, 24: 518-524

[88] Liang LP, Huang J, Fulton R, Pearson-Smith JN, Day BJ, Patel M (2017). Pre-clinical therapeutic development of a series of metalloporphyrins for Parkinson's disease. Toxicol Appl Pharmacol, 326: 34-42

[89] Salvemini D, Wang ZQ, Zweier JL, Samouilov A, Macarthur H, Misko TP, et al. (1999). A nonpeptidyl mimic of superoxide dismutase with therapeutic activity in rats. Science, 286: 304-306

[90] Salvemini D, Riley DP, Cuzzocrea S (2002). SOD mimetics are coming of age. Nat Rev Drug Discov, 1: 367-374

[91] Murphy CK, Fey EG, Watkins BA, Wong V, Rothstein D, Sonis ST (2008). Efficacy of superoxide dismutase mimetic M40403 in attenuating radiation-induced oral mucositis in hamsters. Clin Cancer Res, 14: 4292-4297 